\title{
La taxe pour l'acquisition de titres de spécialiste supplémentaires sera réduite
}

\section{Hänggeli, administrateur responsable du Secrétariat de la formation postgraduée et continue (FPPC)}

Nous avons une bonne et une mauvaise nouvelle à communiquer aux médecins en formation postgraduée. Voici tout d'abord la mauvaise: en raison de la situation financière de la $\mathrm{FMH}$, la Chambre médicale a décidé le 3 mai 2007 de supprimer les restitutions de cotisations accordées pendant les années suivant l'obtention d'un titre de spécialiste. Cette mesure correspond à l'un des quatre modules visant à stabiliser à long terme le budget de la FMH. Et maintenant la bonne nouvelle: les médecins qui obtiennent un deuxième ou un troisième titre après leur premier titre de spécialiste payeront à partir du $1^{\text {er }}$ janvier 2008 Fr. 1500.- au lieu de Fr. 2500.-. Vu la situation financière saine de la FPPC [1], la CFPC a approuvé cette réduction de taxe à l'unanimité.

\section{Rétrospective}

Depuis l'entrée en vigueur de l'accord avec l'UE sur la libre circulation des personnes et de la loi fédérale sur l'exercice des professions médicales (LEPM) au $1^{\text {er }}$ juin 2002, la FMH octroie des titres fédéraux de spécialiste. La suppression de l'affiliation obligatoire à la FMH pour l'obtention et le port d'un titre de spécialiste, intervenue au même moment, nous a contraints à prélever des émoluments couvrant les frais de diplôme en vue de financer le domaine de la formation postgraduée et continue. Faute de quoi, les non-membres auraient financé leur titre de spécialiste sur le dos des membres, ce qui aurait été d'un opportunisme inacceptable. Après quelques années d'observation, nous constatons que la taxe de Fr. 4000.-, fixée en 2001 pour l'octroi d'un titre de spécialiste, est correcte. Avant 2002, la FMH délivrait en moyenne env. 800 titres de spécialiste par année (tab. et fig. 1). Grâce à l'augmentation rapide des titres remis au cours de ces dernières années, le secrétariat FPPC a pu former des réserves qui seront disponibles pour de futurs projets visant à augmenter la qualité de la formation postgraduée.

\section{Solidarité de la FMH} avec la jeune génération de médecins 4000 francs, c'est cher pour un titre de spécialiste. Lors des débats sur la taxe prélevée pour l'octroi d'un titre, la Chambre médicale de 2001 a jugé bon de maintenir une solidarité «intergénérations» entre les membres de la FMH et de créer simultanément une incitation à s'affilier à la FMH. Elle a alors décidé que lors de l'obtention d'un titre de spécialiste, les membres de la FMH se verraient rétrocéder la majeure partie des

\section{Tableau 1}

Titres de formation postgraduée décernés 1996-2006.

\begin{tabular}{lcccr} 
Année & Titres de spécialiste & Formations approfondies & Médecin praticien & Total \\
\hline 1996 & 766 & 0 & 4 & 770 \\
\hline 1997 & 965 & 0 & 49 & 1014 \\
\hline 1998 & 805 & 35 & 88 & 928 \\
\hline 1999 & 873 & 89 & 112 & 1074 \\
\hline 2000 & 805 & 322 & 218 & 1345 \\
\hline 2001 & 776 & 154 & 142 & 1072 \\
\hline 2002 & 1609 & 119 & 473 & 2201 \\
\hline 2003 & 1551 & 472 & 254 & 2277 \\
\hline 2004 & 1140 & 319 & 120 & 1579 \\
\hline 2005 & 1039 & 186 & 135 & 1360 \\
\hline 2006 & 958 & 177 & 134 & 1269
\end{tabular}


Figure 1

Titres de formation postgraduée décernés 1996-2006.

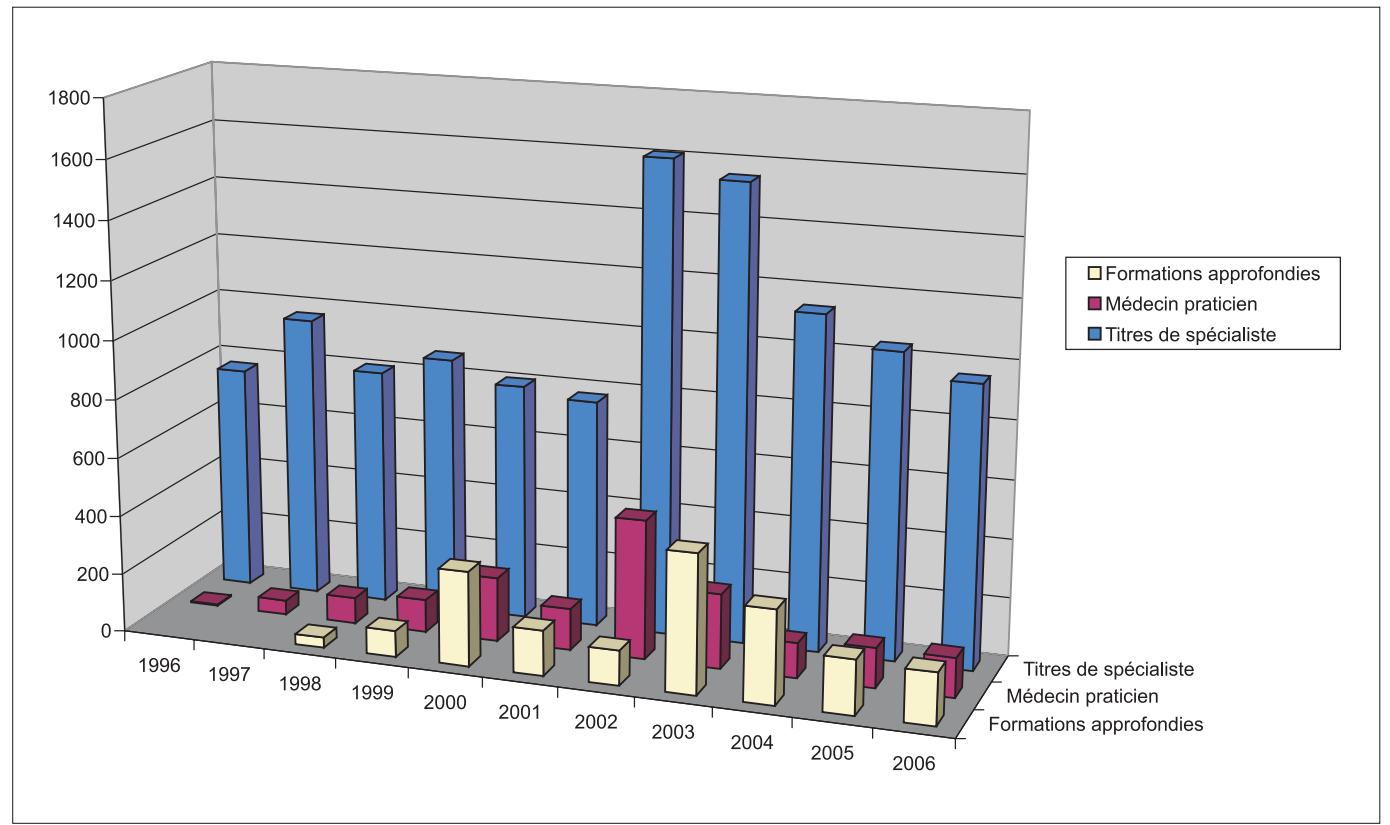

cotisations déjà versées et bénéficieraient en plus d'une réduction de cotisation pour les cinq années à venir. Cette réglementation, selon laquelle le titre de spécialiste ne coûtait en fait plus que Fr. 2000.- au lieu de Fr. 4000.- pour les membres de la FMH, a maintenant été modifiée par la Chambre médicale. Désormais, les membres n'obtiendront plus qu'une restitution de cotisation de Fr. 1000.- au moment de l'acquisition du premier titre de spécialiste. Les médecins ayant obtenu leur diplôme de médecin avant 2007 ne sont pas concernés par cette mesure et bénéficieront d'une restitution de Fr. 2000.- jusqu'en 2013. Selon la décision prise en 2003 , la Chambre médicale continuera, de surcroît, à soutenir des projets de formation postgraduée par des fonds généraux lorsque la situation financière l'exigera.

\section{L'affiliation à la FMH est payante!}

Les médecins qui s'affilient à la FMH dès la première année de formation postgraduée sont gagnants dans tous les cas: d'une part, ils récupèrent une partie de leurs cotisations lors de l'obtention du titre de spécialiste et, d'autre part, tous les renseignements ayant trait à la formation postgraduée (allant de la simple demande à l'établissement d'un plan de formation, etc.) leur sont fournis gratuitement.

\section{$\mathbf{4 0 0 0}$ francs pour un bout de papier?}

Ce reproche souvent entendu est injustifié, dans la mesure où la taxe prélevée ne sert pas seule- ment à examiner la demande de titre et à remettre le document proprement dit. La plus grande partie des dépenses réalisées dans le domaine de la formation postgraduée et continue concerne les tâches liées à la réglementation et à l'amélioration de la qualité de la formation postgraduée. En voici quelques exemples:

- indemnisation de tous les organes et commissions à l'œuvre dans le domaine de la formation postgraduée et continue;

- enquête menée auprès des médecins-assistants sur tout le territoire suisse au sujet de la qualité de leur établissement de formation postgraduée;

- accréditation des programmes de formation postgraduée par le Département fédéral de l'intérieur (DFI);

- certification de tous les établissements de formation postgraduée (hôpitaux, cliniques, divisions, services) en Suisse;

- projets d'évaluation (mini-CEX, auto-évaluation).

Gérer le secrétariat de la formation prégraduée, postgraduée et continue (FPPC) sous forme de centre de services financièrement indépendant permet de séparer clairement l'ensemble de ses dépenses et recettes des comptes généraux de la $\mathrm{FMH}$, et de présenter au public et aux autorités un compte financier transparent pour ce domaine. Les projets et chiffres détaillés figurent dans les rapports d'activité publiés annuellement par le secrétariat FPPC [1]. 


\section{Quelles sont les nouveautés du tarif des émoluments?}

Etant donné que les cotisations ne seront plus restituées pendant les cinq années suivant l'obtention du titre de spécialiste, la CFPC a décidé de réduire la taxe perçue pour l'octroi d'un deuxième ou d'un troisième titre en la faisant passer de Fr. 2500.- à Fr. 1500.-. Cette mesure facilitera l'acquisition de titres de spécialiste supplémentaires. La taxe de Fr. 4000.- facturée pour le premier titre couvre, en tant qu' «émolument de base», l'ensemble des coûts du domaine FPPC. S'agissant des titres supplémentaires, les coûts facturés sont essentiellement ceux de la procédure de remise du titre. Les autres modifications du tarif des émoluments sont plutôt de nature rédactionnelle et ont été effectuées pour des rai- sons de clarté et de précision (entre autres taxes des Commissions d'opposition, prestations assujetties à la TVA, dispositions transitoires).

\section{Perspectives}

Les différents projets qui entreront ces prochaines années en phase de réalisation occasionneront des coûts élevés, mais leur financement sera garanti grâce au tarif des émoluments actuel. Le prélèvement d'une taxe constante au fil des années assure l'égalité de traitement de toutes les personnes obtenant un titre et renforce la confiance dans la FMH. Bien que des augmentations annuelles soient de règle dans le domaine de la santé, il n'en va pas de même au secrétariat FPPC. Et c'est là une autre bonne nouvelle!

\section{Tarif des émoluments}

Le présent tarif des émoluments remplace celui de 2002 [2].
En vertu de l'art. 69 RFP, la CFPC a adopté le 6 septembre 2007 le tarif des émoluments suivant:

\section{Octroi de titres postgrades fédéraux}

Premier titre de spécialiste

Fr. 4000.-

Exclusivement pour les membres de la FMH

Rétrocession sur la cotisation de membre: jusqu'à Fr. 200.- par année d'affiliation pendant 5 ans au maximum lors de l'obtention du premier titre de spécialiste (au maximum Fr. 1000.-).

Deuxième titre de spécialiste et chaque titre suivant Fr. 1500.(les titres étrangers ne sont pas pris en considération)

Taxe pour l'examen de spécialiste ou de formation approfondie La taxe est fixée par la société de discipline médicale concernée.

\section{Octroi de titres de formation postgraduée FMH (exclusivement pour les membres de la FMH)}

Titre de spécialiste (chirurgie de la main et neuropathologie) Fr. 4000.Rétrocession analogue au chiffre 1

Formation approfondie

Fr. 1000.-

Attestation de formation complémentaire

La taxe est fixée par la Société de discipline médicale concernée.

\section{Prestations dans le domaine des titres de formation postgraduée}

Renseignements écrits donnés par le secrétariat FPPC

30 min. à 1 heure

$\begin{array}{ll}\text { Membres } & \text { non-memb } \\ \text { gratuit } & \text { Fr. } 100 .-^{*} \\ \text { gratuit } & \text { Fr. } 200 .-^{*} \\ \text { gratuit } & \text { Fr. } 300 .-^{*}\end{array}$


Etablissement d'un plan de formation postgraduée

\section{Membres non-membres

par la Commission des titres

30 min. à 1 heure

\begin{tabular}{|c|c|}
\hline gratuit & Fr. 200.- ${ }^{*}$ \\
\hline gratuit & Fr. 300.- ${ }^{*}$ \\
\hline gratuit & Fr. 400. ${ }^{\star}$ \\
\hline & Fr. 1000.- \\
\hline & Fr. 150.-* \\
\hline & Fr. 20.- ${ }^{*}$ \\
\hline & Fr. 1000.- ${ }^{\star}$ \\
\hline & Fr. 300.-* \\
\hline
\end{tabular}

\section{1 à 2 heures}

plus de 2 heures

établissement d'une attestation d'équivalence (uniquement pour les porteurs d'un diplôme de médecin non reconnu) établissement d'un duplicata en cas de perte de diplôme facturation d'une authentification certification d'attestations de formation complémentaire gestion des données relatives aux attestations de formatione complémentair

\section{Prestations dans le domaine de la formation continue}

Remise du label «approuvé par la FMH» (art. 7 RFC; cf. Critères

de reconnaissance sur $w w w . f m h . c h)$

première évaluation

Fr. 350.- ${ }^{*}$ session répétée

Fr. $150 .-^{*}$

\section{Prestations dans le domaine des établissements de formation postgraduée}

Taxes de visite d'évaluation

établissements de grande taille

Fr. 5000.-

établissements de petite taille (1 à 3 médecins-assistants)

Fr. 4000.-

Pour les visites concernant plusieurs établissements de formation postgraduée (p. ex. titres de spécialiste et formations approfondies) et/ou un établissement disposant de plusieurs reconnaissances, le Bureau CFPC fixe la taxe de visite en fonction du cas concerné.

\section{Taxes des commissions d'opposition}

Coûts de la procédure d'opposition, selon le temps consacré de

Fr. 500.- à Fr. 1500.-

\section{Dispositions complémentaires et transitoires}

1. La taxe pour l'obtention du titre est facturée lors de l'octroi du titre et doit être réglée dans les 30 jours. Le diplôme n'est remis qu'à la réception du paiement.

2. Les taxes déjà payées pour le titre de formation postgraduée «Médecin praticien» ou pour l'attestation d'équivalence sont prise en compte au moment de l'octroi du titre de spécialiste.

3. La réduction de cotisations accordée pendant les années qui suivent la remise du premier titre de spécialiste est maintenue jusqu'en 2013, toutefois uniquement pour les membres de la FMH qui ont obtenu leur diplôme de médecin avant le $1^{\text {er }}$ janvier 2007.

4. Les médecins qui obtiennent les titres de formation postgraduée FMH «Chirurgie de la main» et «Neuropathologie» selon l'ancien programme de formation postgraduée, versent Fr. 1000.- (somme analogue à celle versée pour l'ancienne formation approfondie). Les pionniers obtiennent le titre selon le nouveau programme.

5. La nouvelle taxe pour un deuxième titre de spécialiste est valable à partir du $1^{\mathrm{er}}$ janvier 2008 (date de reconnaissance).

6. Les dispositions complémentaires et transitoires de l'ancien tarif des émoluments de 2002 restent en vigueur dans la mesure où elles sont encore applicables.

\section{Références}

1 Giger M, Hänggeli C. Rapport d'activité 2006 de la Commission pour la formation postgraduée et continue (CFPC) / Secrétariat FPC. Bull Méd Suisses. 2007;88(23):980-98.

2 Hänggeli C. Comment? 4000 francs pour un titre postgrade fédéral? Bull Méd Suisses. 2002;83(25): 1289-92. 